\title{
AN ANALYTICAL SOLUTION FOR CRITICAL WITHDRAWAL OF LAYERED FLUID THROUGH A LINE SINK IN A POROUS MEDIUM
}

\author{
H. ZHANG ${ }^{1}$, G. C. HOCKING ${ }^{2}$ and D. A. BARRY'
}

(Received 9 August 1995; revised 23 March 1996)

\begin{abstract}
Fluid withdrawn through a line sink from a layered fluid in a vertically confined porous medium is considered. A hodograph method is used to obtain the shape of the interface for a given sink position at the critical flow rate. The analytical solution is compared with a more general numerical solution developed in earlier work. It was found that the surface profiles obtained by the two methods are in close agreement. However, the present work has the advantage that it gives a fully explicit solution.
\end{abstract}

\section{Introduction}

This paper is a companion to an earlier paper [8]. The problem of "water coning" is often encountered in the oil industry when a layer of water underlies a layer of oil. At sufficiently high rates of oil pumping, water is drawn upwards and pumped out together with the oil while the oil-water interface takes the form of a cone. The lowest pumping rate for which both oil and water are pumped out is termed the critical rate. Dagan and Bear [4] presented perturbation solutions for a similar problem in which water is withdrawn from above an immiscible, denser fluid such that the interface is drawn up. Bear and Dagan [2] used the hodograph method to determine the critical flow rate for this occurrence. McCarthy $[5,6]$ solved this problem by assuming a constant potential boundary across the flow field at some distance from the sink in a lateral edge drive model. Recently, Zhang and Hocking [8] employed a model assuming that the flowing layer is confined below by an impermeable boundary, along which it is no longer necessary to satisfy a pressure condition. By allowing the inner edge of this lower boundary to move outward, the effect of the lower boundaries can

\footnotetext{
${ }^{1}$ Department of Environmental Engineering, University of Western Australia, Nedlands, WA 6907, Australia

${ }^{2}$ School of Mathematics and Physical Sciences, Murdoch University, Murdoch, WA 6150, Australia

(C) Australian Mathematical Society, 1997, Serial-fee code 0334-2700/97
} 
be gauged. This model could also be considered as a problem in which a layer of oil is trapped between two impermeable rock strata exposed to a small aquifer near the well site. A nonlinear integral equation for the angle between the interface and horizontal was derived and solved numerically.
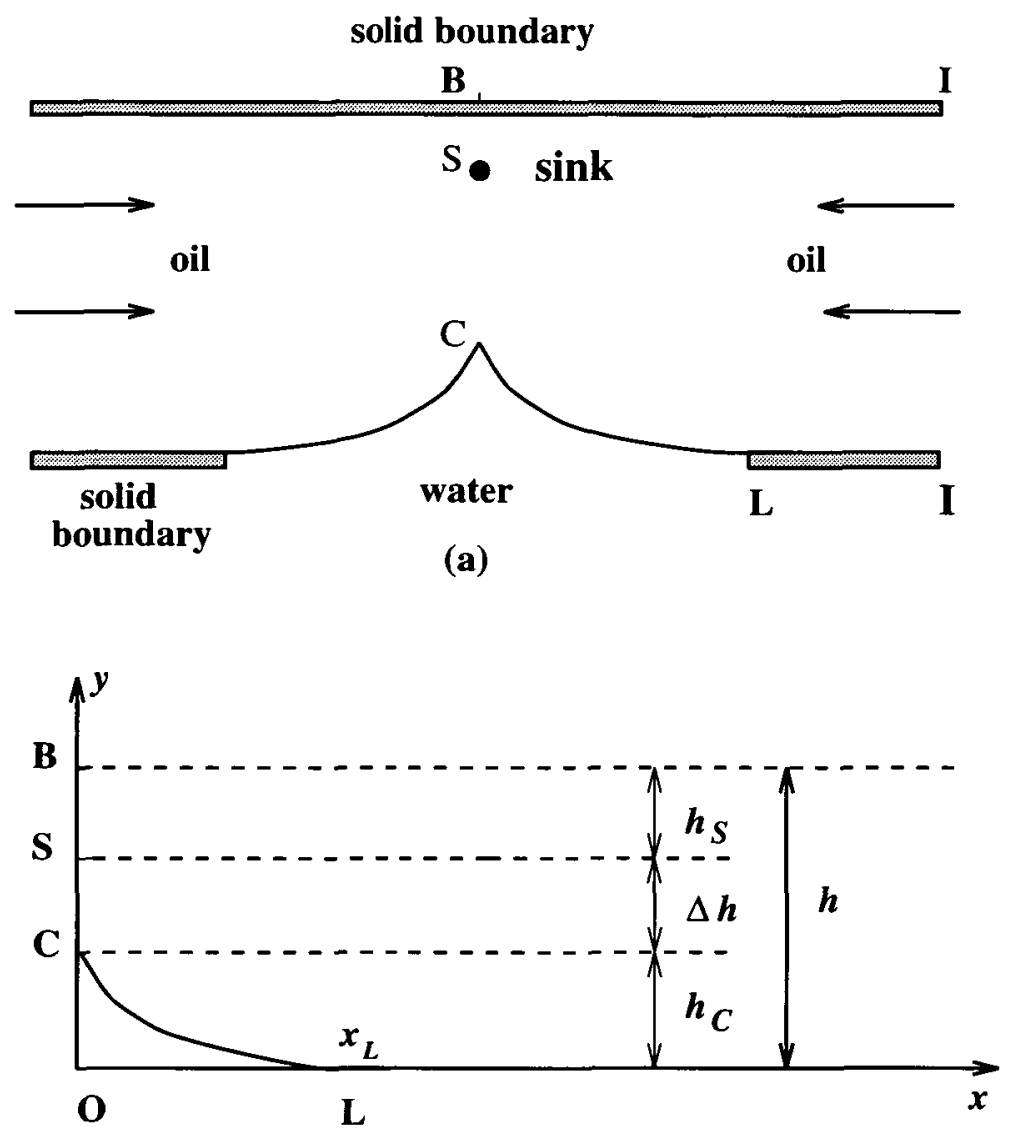

(b)

FIGURE 1. Configuration of the confined oil reservoir: (a) physical plane (b) z-plane.

In this study, an oil reservoir consisting of an oil layer confined above by an impermeable boundary is considered. The physical plane is shown in Figure 1(a). A line sink (S) is located in the oil layer. BI is the top impermeable boundary. LI is the bottom solid boundary. $\mathrm{C}$ is the cusp point of the interface. A hodograph method is used to solve the critical-rate coning problem. It has the major advantage that it is an 
exact analytic solution, whereas the previous solution [8] is numerical.

\section{Problem formulation}

Consider a homogeneous and isotropic porous medium of constant permeability $k$ beneath a boundary of impermeable rock. A layer of oil of depth $h$ exists above an unbounded region of water, as shown in Figure 1. A line sink is located at a distance, $h_{S}$, beneath the impermeable boundary. The coning height is $h_{C}$. The horizontal distance between the sink and each bottom boundary is $x_{L}$. The sink produces a total flux $Q$ per unit time.

It is convenient to define dimensionless variables. In the following analysis, we use $y^{*}=y / h, x^{*}=x / h, h_{S}^{*}=h_{S} / h, h_{C}^{*}=h_{C} / h$ and $\Delta h^{*}=\Delta h / h$. It is apparent that solutions to this problem are dependent on the dimensionless parameters $h_{S}^{*}, x_{L}^{*}$ and $c=K h / \bar{Q}$, where $K=k\left(\gamma_{w}-\gamma_{o}\right) \gamma_{o}$, and $\gamma_{w}, \gamma_{o}$ are the specific gravity of water and oil, respectively.

Note that the dimensionless flow rate is $1 / c$. Using complex variables, let the physical plane correspond to the $z$-plane (see Figure 1(b)), where $z=x+i y$. The origin is located directly beneath the sink at the level of the lower solid boundary. Darcy's equation for flow through porous media is

$$
\mathbf{q}=-\nabla \phi,
$$

where $\mathbf{q}$ is the specific discharge vector and $\phi$ is the velocity potential function. The velocity components $u$ and $v$ are related to $\phi$ by

$$
u=-\frac{\partial \phi}{\partial x}, \quad v=-\frac{\partial \phi}{\partial y} .
$$

The assumptions that the fluid is incompressible and that Darcy's law is valid lead to a problem in which Laplace's equation,

$$
\nabla^{2} \phi=0,
$$

must be solved subject to appropriate boundary conditions.

Within the flow domain, there is no flow across the solid boundaries and interface, a condition which can be described by $\mathbf{q} \cdot \mathbf{n}=0$, where $\mathbf{n}$ is in the direction of the outward normal. The lower fluid is stationary, and the entire stationary fluid region is assumed to be at a constant potential. The free surface condition along the stationary interface between the moving fluid (oil, $\gamma_{o}$ ) and a stationary fluid (water, $\gamma_{w}$ ) is [1]

$$
u^{2}+v^{2}-K v=0 \text {. }
$$

At the critical flow rate, the velocity components at $\mathrm{C}$ are $u=0$ and $v=K$, and the velocity at $\mathrm{L}$ is zero. The flow rate is uniform as $x \rightarrow \pm \infty$. 


\section{Hodograph method and results}

Hodograph mapping is very useful when the boundaries consist of planes and a curved free surface, as the hodograph is easily obtained from which the flow problem may be solved analytically. Although the position of the free surface is unknown, its hodograph is known. From (2.3), we find that in the critical flow case the free surface is a circular arc in the hodograph plane. The hodograph method can be employed to solve this problem in a manner similar to that used by Yih [7] for the case of a free surface flow without a cusp.

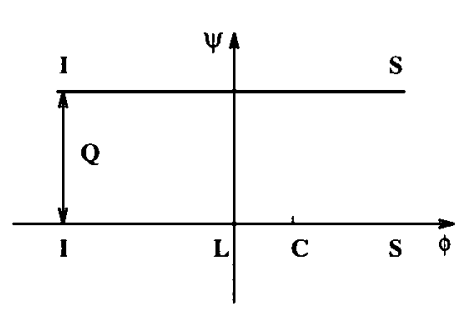

(a)

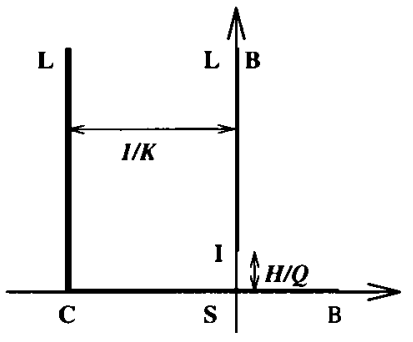

(b)

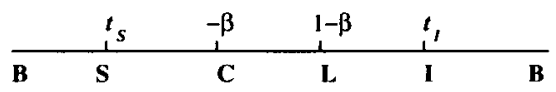

(c)

FIGURE 2. Mapped planes used in the analytical method: (a) $w$-plane (b) $\zeta$-plane (c) $t$-plane.

Let $\omega=\phi(x, y)+i \psi(x, y)$ be the complex potential. The transformations

$$
\begin{gathered}
\zeta=i \frac{d z}{d \omega} \\
\zeta=-2 M\left(\sqrt{t+\beta}+t_{I} \tanh ^{-1} \sqrt{t+\beta}\right)-N-\frac{1}{K},
\end{gathered}
$$

require that $\zeta_{L}, \zeta_{I}, \zeta_{S}$ and $\zeta_{C}=-1 / K$ should be transferred to $t_{L}=1-\beta, t_{l}, t_{S}$ and $t_{C}=\beta$, respectively. The mapped planes used in this procedure are shown in Figure 2. Substitution of $\zeta_{C}=-1 / K$ and $t_{C}=-\beta$ into (3.2) gives $N=0$. The imaginary part of $\tanh ^{-1} \sqrt{t+\beta}$ for a positive $t$ is $-i \pi / 2$. When $t$ changes from $0^{-}$ to $0^{+}, \zeta$ is changed from $1 / K$ to 0 . So $M$ can be determined as $M=-i /\left(t_{l} K \pi\right)$. 
The transformation between $\zeta$ and $t$ can finally be written as

$$
\zeta=\frac{2 i}{t_{l} K \pi}\left(\sqrt{t+\beta}+t_{l} \tanh ^{-1} \sqrt{t+\beta}\right)-\frac{1}{K} .
$$

Upon substituting $\zeta=i h / Q$ and $\zeta_{S}=0$ into (3.3) and choosing the negative imaginary part of the square root, we find that $t_{I}$ can be determined from

$$
\frac{c \pi}{2}=\frac{\sqrt{t_{l}+\beta}}{t_{l}}+\operatorname{coth}^{-1} \sqrt{t_{l}+\beta}, t_{l}>0,
$$

while

$$
\frac{2}{t_{1} \pi} \sqrt{-t_{S}-\beta}+\frac{2}{\pi} \tan ^{-1} \sqrt{-t_{S}-\beta}-1=0, t_{S}<-\beta,
$$

can be used to determine $t_{s}$.

The transformation

$$
\omega=\frac{Q}{\pi} \ln \frac{t_{I}-t}{t-t_{S}}
$$

is applied as well. Because this mapping is conformal, only the principal value of $\tan ^{-1} \sqrt{-t_{S}-\beta}$ in (3.5) is taken. Hence $z$ can be expressed in terms of $t$ using (3.1) and (3.3):

$$
d z=\frac{1}{c \pi}\left(\frac{2}{t_{l} \pi} \sqrt{t+1}+\frac{2}{\pi} \tanh ^{-1} \sqrt{t+1+i}\right)\left(\frac{1}{t-t_{I}}-\frac{1}{t-t_{S}}\right) d t .
$$

The shape of the interface is then described by

$$
\begin{gathered}
x^{*}=\int_{-\beta}^{t}-\frac{2}{c \pi^{2}}\left(\frac{\sqrt{a+1}}{t_{I}}+\tanh ^{-1} \sqrt{a+1}\right)\left(\frac{1}{a-t_{I}}-\frac{1}{a-t_{S}}\right) d a \\
y^{*}=\frac{1}{c \pi}\left(\ln \frac{t_{1}-t}{t-t_{S}}-\ln \frac{t_{I}-1+\beta}{1-\beta-t_{S}}\right),
\end{gathered}
$$

for $-\beta \leq t \leq 1-\beta$. The equation for $x^{*}$ cannot be evaluated analytically, but can be computed numerically using standard techniques.

Figure 3 shows the interface shapes calculated for various values of $c$, compared . with those obtained by the numerical method developed in [8]. It can be seen that they are in good agreement. The sink position $h_{S}^{*}$ can also be calculated by integrating the imaginary part of (3.7) for $-\infty<t \leq t_{S}$ as

$$
\int_{t_{S}}^{-\infty}-\frac{1}{c \pi}\left(\frac{2}{t_{l} \pi} \sqrt{-t-\beta}+\frac{2}{\pi} \tan ^{-1} \sqrt{-t-\beta}-1\right)\left(\frac{1}{t-t_{l}}-\frac{1}{t-t_{S}}\right) d t=h_{S}^{*} .
$$




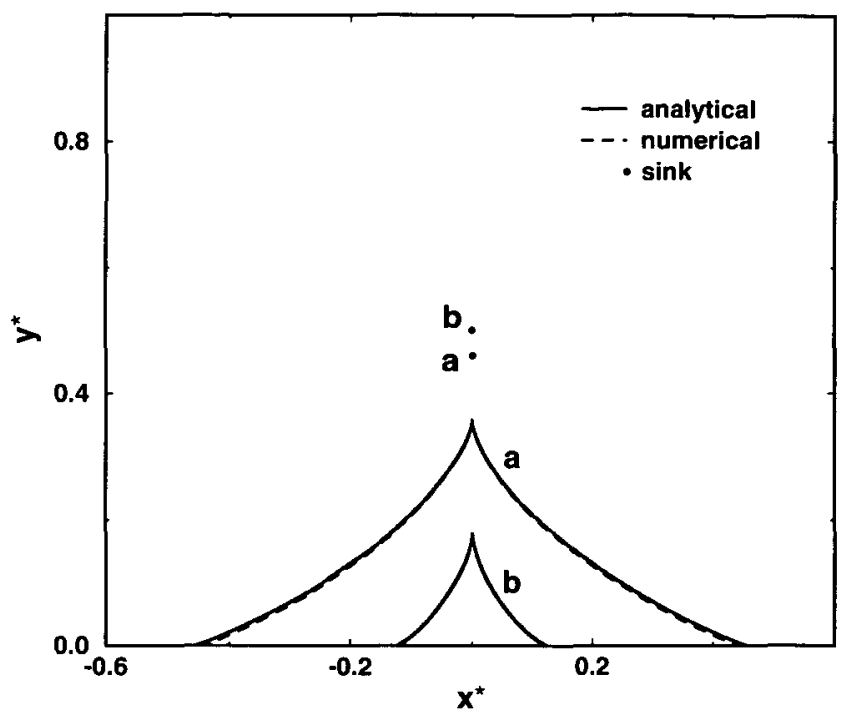

FIGURE 3. The shapes of the interface calculated using the analytical method $(\beta=1)$ compared with the numerical method [9] for the cases of (a) $c=2.0, h_{S}^{*}=0.46$ and $x_{L}^{*}=0.46$, (b) $c=0.5, h_{S}^{*}=0.50$ and $x_{L}^{*}=0.13$.

Because of the condition (3.5), the integrand of (3.10) is nonsingular as $t \rightarrow t_{s}$. It can be integrated numerically using for example, the Maple software package [3]. Obviously, the coning height $h_{C}^{*}$ can be calculated using (3.9) as

$$
h_{C}^{*}=\frac{1}{c \pi}\left(\ln \frac{t_{I}+\beta}{-\beta-t_{S}}-\ln \frac{t_{I}-1+\beta}{1-\beta-t_{S}}\right) .
$$

Varying the parameters $c$ and $\beta$, the solutions for all locations of the sink and the bottom boundary can be obtained. Using (3.4) and (3.5), we can compute a regular expansion in powers of small $c$ :

$$
t_{I}=\frac{16}{\pi^{2} c^{2}}+\frac{1}{3}+O\left(c^{2}\right), \quad t_{S}=-\frac{16}{\pi^{2} c^{2}}-\beta+O\left(c^{2}\right) .
$$

If the expansions (3.12) are substituted into (3.10), it is found that $h_{S}^{*} \rightarrow 1 / 2$ as $c \rightarrow 0$.

For large $c$, the expansions can be derived in powers of $1 / c$ in a similar procedure, but they depend on $\beta$. In that case, if $\beta \leq 1$, they are

$$
\begin{gathered}
t_{I}=\frac{2 \sqrt{\beta}}{\pi} \frac{1}{c}+\frac{2+2 \ln \left(\frac{\sqrt{\beta}+1}{\sqrt{\beta}-1}\right)}{\pi^{2}}+O\left(\frac{1}{c^{3}}\right), \\
t_{S}=-\beta-\beta \frac{1}{c^{2}}+O\left(\frac{1}{c^{4}}\right) .
\end{gathered}
$$


As $c \rightarrow \infty, t_{l} \rightarrow 0, t_{S} \rightarrow-\beta$ and $h_{S}^{*} \rightarrow l$. For $\beta<l$, let $t_{l}=1-\beta+\varepsilon$. The parameter $\varepsilon$ is then related to $c$ as

$$
\frac{1}{c}=\frac{\pi}{\ln 4-\ln \varepsilon+\frac{2}{1-\beta}}+\frac{\frac{2 \pi}{(1-\beta)^{2}}-\frac{\pi}{1-\beta}-\frac{\pi}{2}}{\left(\ln 4-\ln \varepsilon+\frac{2}{1-\beta}\right)^{2}} \varepsilon+O\left(\varepsilon^{2}\right) .
$$

From the dominant term of (3.15), the expression

$$
\varepsilon=4 \exp \left(\frac{2}{1-\beta}-c \pi\right)
$$

can be obtained. If $\theta=\pi / 2-\tan ^{-1} \sqrt{-t-\beta}$ is substituted into (3.5), then $t_{S}$ can be found using (3.16) and

$$
\frac{2}{1-\beta+\varepsilon} \theta_{S}=\cot \theta_{S}, \quad t_{S}=-\beta-\cot ^{2} \theta_{S} .
$$

As $c \rightarrow \infty, t_{l} \rightarrow 1-\beta, t_{S}$ depends on $\beta$ and $h_{S}^{*} \rightarrow 0$.

The results of the hodograph method establish a relationship between the locations of the sink, the bottom boundary and the value of the critical flow rate. To determine this relationship, the flow rate $1 / c$, and $\beta$ were varied and the resulting flows computed. The position of the cone, $\mathrm{C}$, can be calculated from (3.11), and the location of the bottom boundary can be obtained by integrating (3.8) from $-\beta$ to $1-\beta$. The sink location can be calculated by integrating (3.10).

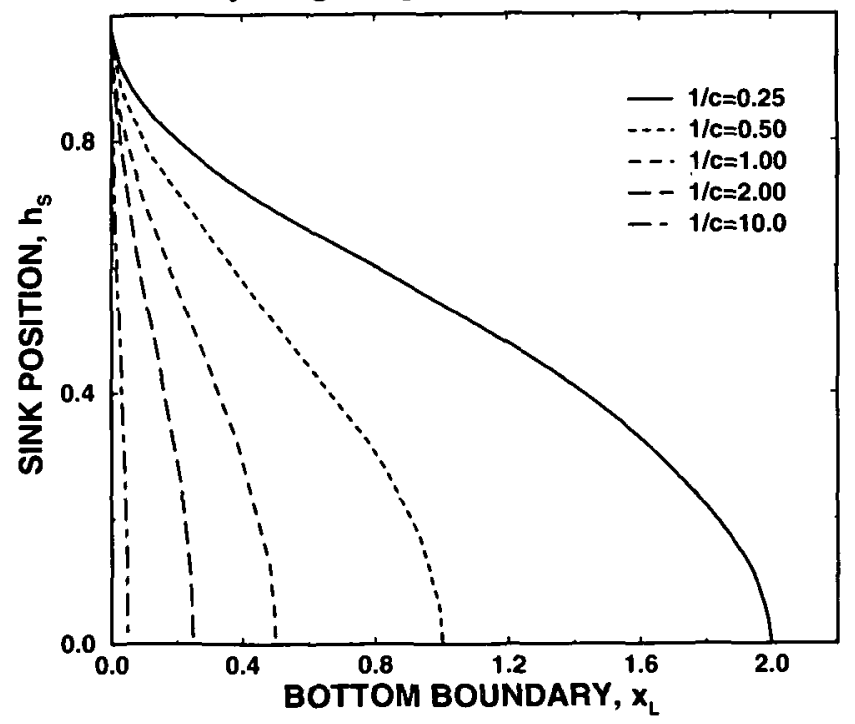

FIGURE 4. The relationship between the flow rate $1 / c$, the sink position $h_{S}^{*}$ and bottom boundary location $x_{L}^{*}$. 
Figure 4 is the major result of this paper. It shows the relationship between $h_{S}^{*}, x_{L}^{*}$ and $1 / c$. If the sink position is fixed, the critical flow rate decreases as the bottom boundary moves outwards, suggesting that for a long thin oil region with no such lower boundary, the critical flow value may be very small. Conversely, it is clear that a very high flow rate is required to induce coning when the gap in the low boundary is very small. In fact as $x_{L}^{*} \rightarrow 0$, the critical flow rate, $1 / c$ increases without bound. Physically, this suggests that one must withdraw rapidly to "pull" the interface away from the lower boundary.

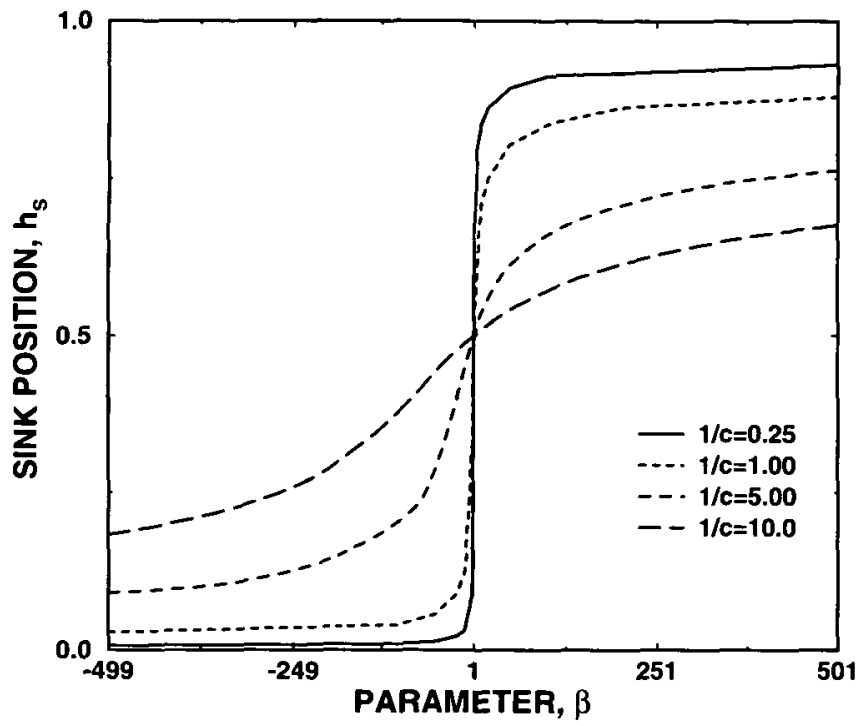

FIGURE 5. The relationship between the flow rate $1 / c$, the sink position $h_{S}^{*}$ and the parameter $\beta$.

Figure 5 shows the relationship between the parameters $\beta, c$ and the sink location $h_{S}^{*}$. It can be seen that the sink is located at $0<h_{S}^{*}<1 / 2$ if $\beta<1$, and is located at $1 / 2<h_{S}^{*}<1$ if $\beta \geq l$, for all flow rates.

The relationship between parameters $\beta, c$ and bottom boundary location $x_{L}^{*}$ is shown in Figure 6. The interesting feature of this plot is that when $\beta \geq 1$, i.e. $h_{S}^{*}>1 / 2, x_{L}^{*}$ is very small. This implies, given the results in Figure 5, that as the sink gets closer to the level of the bottom boundary, very small flow rates will induce coning. Again this is consistent with physical intuition.

\section{Conclusion}

In this paper analytical solutions for the shape of an oil-water interface induced by flow into a line sink have been obtained. The hodograph method has allowed us to obtain analytical solutions for any sink or bottom boundary locations. A cusp solution corresponding to critical flow can be obtained for each such geometry. The interface shape and the sink location were calculated for various critical flow rates. 


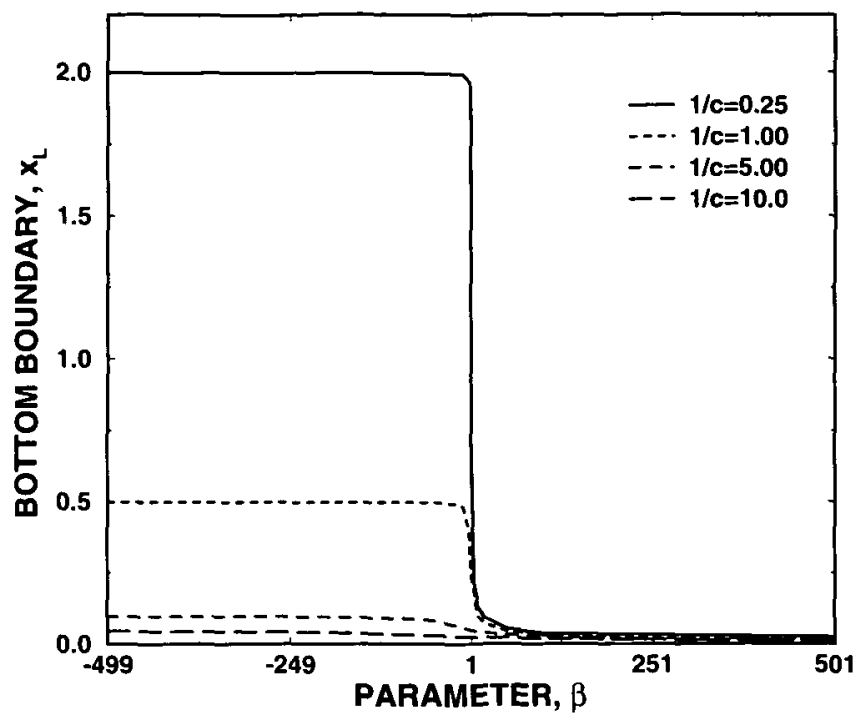

FIGURE 6. The relationship between the flow rate $1 / c$, the bottom boundary location $x_{L}^{*}$ and the parameter $\beta$.

A relationship between these parameters was obtained. Over the range of parameter values at which the solutions were obtained, there was good agreement with both the interface shapes and with the critical coning heights for different sink locations obtained using a numerical method [8].

\section{Acknowledgment}

The authors would like to thank L. K. Forbes for his suggestion to extend the earlier work in this way, and the two anonymous referees whose suggestions resulted in a significant improvement in this work.

\section{References}

[1] J. Bear, Dynamics of fluids in porous media (American Elsevier, New York, 1972).

[2] J. Bear and G. Dagan, "Some exact solutions of interface problems by means of the hodograph method", J. Geophys. Res. 69 (1964) 1563-1572.

[3] B. W. Char, K. O. Geddes, G. H. Gonnet, B. L. Leong, M. B. Monagan and S. M. Watt, Maple library reference manual (Springer-Verlag, New York, 1991).

[4] G. Dagan and J. Bear, "Solving the problem of local interface upconing in a coastal aquifer by the method of small perturbations", J. IAHR 1 (1968) 15-44.

[5] J. F. McCarthy, "Gas and water cresting towards horizontal wells", J. Austral. Math. Soc. Ser. B 35 (1993) 174-197.

[6] J. F. McCarthy, "Improved model of water cresting", J. Austral. Math. Soc. Ser. B 35 (1993) 207-222.

[7] C. Yih, Stratified flows (Academic Press, 1980).

[8] H. Zhang and G. C. Hocking, "Withdrawal of layered fluid through a line sink in a porous medium", J. Austral. Math. Soc. Ser. B 38 (1996) 240-254. 